\title{
Evolution of the number of accreting white dwarfs with shell nuclear burning and of occurrence rate of SN Ia
}

\author{
L.R. YUNGELSON* \\ Institute of Astronomy, Moscow, Russia
}

\begin{abstract}
We analyze temporal evolution of the number of accreting white dwarfs with shell hydrogen burning in semidetached and detached binaries. We consider a stellar system in which star formation lasts for 10 Gyr with a constant rate, as well as a system in which the same amount of stars is formed in a single burst lasting for 1 Gyr. Evolution of the number of white dwarfs is confronted to the evolution of occurrence rate of events that usually are identified with SN Ia or accretion-induced collapses, i. e. with accumulation of Chandrasekhar mass by a white dwarf or a merger of a pair of CO white dwarfs with total mass not lower than the Chandrasekhar one. In the systems with a burst of star formation, at $t=10^{10} \mathrm{yr}$, observed supersoft X-ray sources, most probably, are not precursors of SN Ia. The same is true for an overwhelming majority of the sources in the systems with constant star formation rate. In the systems of both kinds mergers of white dwarfs is the dominant SN Ia scenario. In symbiotic binaries, accreting CO-dwarfs do not accumulate enough mass for SN Ia explosion, while ONeMg-dwarfs finish their evolution by an accretion-induced collapse with formation of a neutron star.
\end{abstract}

*email: lry@inasan.ru 


\section{Introduction}

The nature of progenitors of SN Ia is still unclear. There exists a consensus that exploding objects are white dwarfs (WD) of Chandrasekhar $\left(M_{C h}\right)$ or larger mass, but the way of matter accumulation is still discussed. Historically, three main scenarios were suggested: (i) accretion from the wind in a symbiotic binary (Truran and Cameron, 1971), (ii) accretion in a semidetached binary ("Single-degenerate scenario" (SD), Whelan and Iben, 1973), (iii) merger of components in a binary WD with total mass $\gtrsim M_{C h}$ ("Double-degenerate scenario" (DD), Webbink, 1984; Iben and Tutukov, 1984). Knowledge of SN Ia scenarios is of immense importance for cosmology, since in scenarios (i) and (ii) the mass of exploding object is $M_{C h}$ and the supernova may, most probably, be considered as a standard candle. In scenario (iii), after several tens of Myr after beginning of star formation first explode merging pairs with total mass sufficiently larger than $M_{C h}$, while explosions of the pairs with $M_{t o t}$ close to $M_{C h}$ start only $\sim 10^{9}$ yr later (Tutukov and Yungelson, 1994; Bogomazov and Tutukov, 2009).

In scenarios (i) and (ii) the binary passes through a stage in which the first-formed WD accretes the matter from companion. It is assumed that the (quasi)stationary hydrogen and helium burning at the surface of a WD allows to accumulate $M_{C h}$. In scenario (3), a similar stage precedes formation of the second WD in the system.

Following van den Heuvel et al. (1992), WD with (quasi)stationary burning at the surface are identified with supersoft X-ray sources (SSS). Recently, Di Stefano (2010a,b) called attention to the fact that in the galaxies of different morphological types there exists a significant (up to 2 orders of magnitude) deficit of SSS as compared with expectations based on the SN Ia rate. Independently, to the same conclusion came Gilfanov and Bogdán (2010), based on the study of the luminosity of elliptical galaxies in the supersoft X-ray range.

In this note we study evolution of the numbers of accreting WD and potential SN Ia progenitors. Computations were carried out for two cases of star formation history: (i) assuming a constant star formation rate for $10^{10} \mathrm{yr}$ and (ii) for a case when the same amount of mass as in the previous case is converted into stars in $10^{9} \mathrm{yr}$. Thus, our computations exemplify idealized spiral and elliptical galaxies. It is shown that at the age of $10 \mathrm{Gyr}$ all systems with accreting WD in elliptical galaxies and their overwhelming majority in spiral galaxies are not precursors of SN Ia. We confirm suggestion that the merger of WD is the main channel for SN Ia. We show that in symbiotic binaries, most probably, only oxygen-neon-magnesium WD are able to accumulate $M_{C h}$; then they collapse with formation of neutron stars and do not produce SN $\mathrm{Ia}$.

\section{The method of computations}

Earlier, we computed populations of accreting WD, for instance, in our studies of symbiotic stars (Yungelson et al., 1995; Lü et al., 2006), supersoft X-ray sources (Yungelson et al., 1996; Fedorova et al., 2004), distributions of supernovae over redshift (Yungelson and Livio, 1998, 2000). The main assumptions of our population synthesis code are described in the abovementioned papers and in Tutukov and Yungelson (2002)2. Let note only several details.

Like in our previous studies it is assumed that all stars are born in binaries and star

\footnotetext{
${ }^{1}$ We make a caveat that we use the term "SN Ia" for situations when carbon-oxygen WD accumulates $M_{C h}$ despite the fact that, strictly speaking, model computations of explosions of WD are still unable to reproduce all features of observed SN Ia.

${ }^{2}$ The only exception is the paper by Lü et al. (2006) in which we used modified by us Hurley et al. (2002) code.
} 
formation rate is defined as $\left(\right.$ in $\left.\mathrm{yr}^{-1}\right)$

$$
\frac{d N}{d t}=0.2 d(\log a) \frac{d M_{1}}{M_{1}^{2.5}} f(q) d q,
$$

where $M_{1}$ is the mass of the primary component, $f(q)$ is the distribution of stars over mass-ratio of components $q=M_{2} / M_{1}$ normalized to 1 . Distribution $f(q)$ is

$$
f(q)= \begin{cases}1 & \text { for close binary stars } \\ 0.1055 q^{-2.5} & \text { for wide binaries with } 1 \geq q \geq 0.3 \\ 2.14 & \text { for wide binaries with } 0.3>q \geq 0\end{cases}
$$

Star formation rate is normalized to the formation of one binary with $M_{1} \geq 0.8 M_{\odot}$ per yr. The range of semi-major axes of orbits is $6\left(M_{1} / M_{\odot}\right)^{1 / 3} \leq a / R_{\odot} \leq 10^{6}$ (Kraicheva et al., 1981; Vereshchagin et al., 1988). Single stars in the model are merger products or components of binaries disrupted by supernovae explosions. For the minimum mass of components of binaries $0.1 M_{\odot}$, Eqs. (11) and (2) give model star formation rate $\simeq 8 M_{\odot}$ per yr. This value is consistent with the observational estimates of the current star formation rate in the Galactic disk (Gilmore, 2001). Assuming that the age of model galaxies is $10^{10} \mathrm{yr}$, for the constant rate of star formation one obtains that the total mass of stars processed in the stars is close to $810^{10} M_{\odot}$, comparable to the observational estimates of the mass of Galactic disk (Dehnen and Binney, 1998). The mass of model galaxies and formation rates and numbers of different systems rendered below may be rescaled by a simple change of numerical coefficient in Eq. 1.

Taking into account the specifics of the evolution of close binaries, we assume in the model that the minimum mass of the components of close binaries that explode as core-collapse supernovae is $11.5 M_{\odot}$; in wide systems this threshold is $10 M_{\odot}$. Then the occurrence rate of SN II and SN b,c is close to 1 per $40 \mathrm{yr}$. This estimate does not contradict observational estimate of the Galactic formation rate of pulsars - $(0.9-1.9)$ per $100 \mathrm{yr}$ (Vranesevic et al., 2004). Occurrence rate of SN Ia is discussed below.

Stellar wind mass-loss was computed using Reamers' formula $\dot{M}=4 \times$ $10^{-12} \eta\left(R / R_{\odot}\right)\left(L / L_{\odot}\right)\left(M / M_{\odot}\right)^{-1} \mathrm{M}_{\odot} \mathrm{yr}^{-1}$ with variable parameter $\eta$ (see Yungelson et al., 1995). For computation of stellar wind accretion rate standard Bondi-Hoyle formalism was applied (Bondi, 1952).

In calculations of the numbers of accreting WD with surface nuclear burning we considered both objects with stationary and unstable burning (see Yungelson et al.1996). We considered both possibility of accumulation of the matter and possibility of erosion of WD due to unstable nuclear burning. Conditions for unstable burning of hydrogen were taken from Prialnik and Kovetz (1995), while for helium - from Iben and Tutukov (1996).

Let list several differences to the paper of Yungelson and Livio (1996) which was immediately aimed at the study of WD with nuclear burning which were identified in that paper with supersoft X-ray sources.

(i) Following Kato and Hachisu (1994), in the present paper we assumed that in the case of accretion rate $|\dot{M}| \leq 10^{-4} \mathrm{M}_{\odot} \mathrm{yr}^{-1}$ and larger than the upper limit of the rate of stationary hydrogen burning $\log \left(\left|\dot{M}_{\max }\right|\right) \approx-9.31+4.12 M_{w d}-1.42 M_{w d}^{2}\left(\right.$ in $\left.\mathrm{M}_{\odot} \mathrm{yr}^{-1}\right)$, excess of the matter leaves the system via optically thick stellar wind from WD and takes away specific angular momentum of WD. Under this assumption, the maximum mass ratio of components which still allows stable mass-exchange increases from $q \approx 0.78$ to $q \approx 1.15$. More, mass-exchange in the thermal time scale of the donor becomes possible, while in the earlier versions of computations it was assumed that in all such systems a common envelope forms. Computational algorithm corresponding to these assumptions is described in detail by Yungelson and Livio (1998). 
(ii) We took into account stabilizing effect of relatively massive cores on dynamical stability of mass loss by red giants (Hjellming and Webbink, 1987).

(iii) At difference to population synthesis studies mentioned above, for the estimate of the variation of separation of components in the first unstable mass-exchange episode in the systems with comparable masses of components, we applied so-called $\gamma$-formalism, based on the balance of angular momentum (Nelemans et al., 2000). In this case, separation of components varies only insignificantly in the course of mass exchange. In all other cases of unstable mass-exchange Webbink's (1984) equation for common envelopes was applied, using fixed value of the product of common envelope parameter $\alpha_{c e}$ and stellar structure parameter $\lambda$. Results presented below were obtained for $\gamma=1.5$ and $\alpha_{c e} \lambda=2$, since this combination of parameters allows to explain the characteristics of observed close binary WD and other low-mass detached systems with WD components (Nelemans et al., 2001; Nelemans and Tout 2005).

The result of the above-mentioned changes in the code is that substantially larger fraction of binaries than computed before passes trough the stage of stationary mass exchange instead of forming common envelopes and the number of systems with subgiant donors increases by about an order of magnitude compared to the number obtained in Yungelson an et al., (1996). In symbiotic binaries, the number of WD with stationary burning increases by a factor $\simeq 2.5$.

We did not consider the possibility of stabilization of mass exchange due to additional mass and momentum loss from the system due to stripping of red giant by WD wind (Hachisu et al., 1999). If the latter assumption is correct, one would expect the presence of a significant amount of circumstellar matter in the vicinity of SN Ia progenitors. However, existing observational data indicate the presence of hydrogen in the vicinity of only one of the known "normal" SN Ia - SN 2006X (Patat et al., 2007). Energy release due to collision of the supernova ejectum with its companion star should produce a conspicuous kink in the early light curve lasting for $\sim$ day following the explosion, especially notable in UV. Existing observational data apparently already now allow to exclude red-giant companions with $\sim 1 M_{\odot}$ and distances between components $\sim 10^{13} \mathrm{~cm}$ (Kasen, 2010). More, for the desired effect on the SN Ia rate, the efficiency of the stripping, which is a parameter of the problem, needs to have an unlikely value very close to 1 .

We did not consider possible effect of magnetic fields of WD on the accretion regime, which, most probably, only diminishes the efficiency of accretion (by about 30\%, Lipunov and Postnov (1988)).

\section{Results and discussion}

In the present paper, we did not aim at a detailed study of relation between the number of accreting WD and SN Ia for numerous variations of the parameters of population synthesis, but limited ourselves by presenting an illustratory model which, to our opinion, clearly enough exemplifies this relation.

Results of computations for two model galaxies are presented in Fig. 1,

\section{Close binary white dwarfs and semidetached binaries}

Yungelson et al.(1996) have shown that the population of semidetached systems with hydrogen-burning accreting WD is dominated by the systems with subgiant donors. Therefore, we restricted ourselves by consideration of these systems only.

In star-formation model (i) at the age $t=10^{10} \mathrm{yr}$ occurrence rate of the merger of WD with total mass exceeding $M_{C h}$ is $3.2 \times 10^{-3} \mathrm{yr}^{-1}$ in agreement with the rate of SN Ia in Sbc-type galaxies (Cappellaro et al., 1999). In case (ii) this rate is $4.5 \times 10^{-4} \mathrm{yr}^{-1}$. Thus, if merger of WD is identified with SN Ia, the ratio of events in two cases per unit mass is $\simeq 7$. This is close to the ratio $\lesssim 5$ for $\mathrm{E} / \mathrm{S} 0$ and $\mathrm{Sbc} / \mathrm{d}$ galaxies found by Mannucci et 

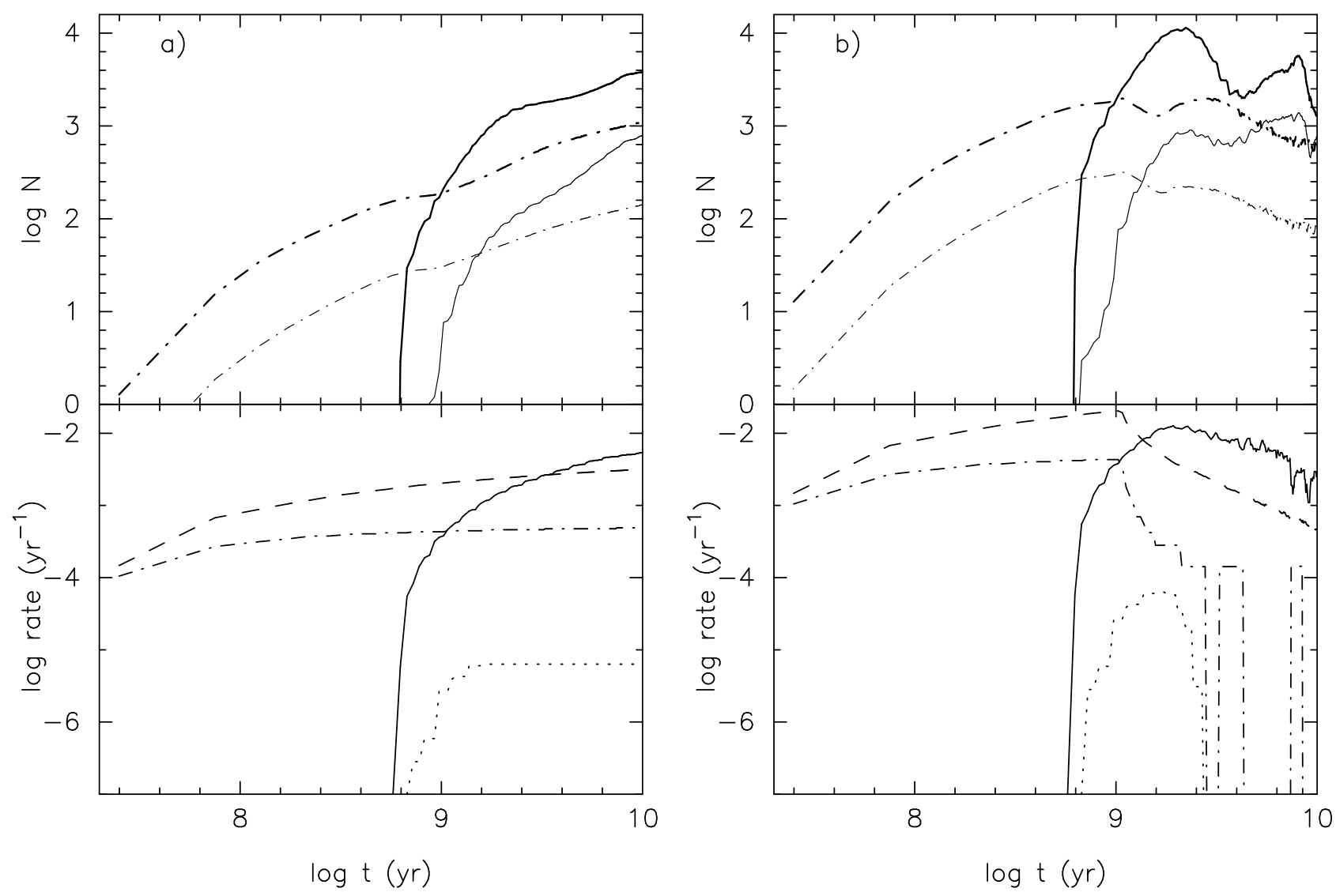

Figure 1. Evolution of the formation rate of accreting WD with surface hydrogen burning, of occurrence rate of SN Ia and accretion-induced collapses and of the number of binaries associated with them. a) a system with star formation rate constant for $10^{10} \mathrm{yr}$. In the lower panel dashed line shows occurrence rate of SN Ia in double-degenerate scenario, dotted line shows occurrence rate of SN Ia in singledegenerate scenario. Dash-dot line shows the rate of AICs in symbiotic systems, solid line - the rate of formation of semidetached systems with hydrogen-burning WD. In the upper panel thick solid line shows the number of semidetached systems with subgiant donors in which WD burn hydrogen stationary, thin solid line - the number of the system of the same type with WD burning hydrogen unstably. Thick and thin dash-dot lines show the numbers of symbiotic stars with stationary and non-stationary hydrogen-burning WD, respectively. b) - the same as in (a) but for a system with a $10^{9} \mathrm{yr}$ long star-formation burst that produced the same mass of stars as in case (a).

al. (2005). More fine tuning of this ratio makes no sense for illustrative calculation. In case (i) occurrence rate of accumulations of $M_{C h}$ in semidetached systems at the age $t=10^{10}$ is $6 \times 10^{-6} \mathrm{yr}^{-1}$. Qualitatively, the ratio of mergers and $M_{C h}$ accumulations does not differ from the ratio found in our earlier computations, where it never exceeded $\sim 0.1$. The main reason for the low efficiency of the SD-scenario is, in our case, low efficiency of matter accumulation: combinations of masses of WD and accretion rates is favorable for unstable hydrogen burning and loss of most of accreted hydrogen in Novae explosions.

Formation rate of close binary WD reflects SFR, the mergers start several tens of Myr after beginning of star formation, when the binaries that had ZAMS masses of components close to $10 M_{\odot}$ start to form the pairs of WD. More specifically, time-delay of SN Ia in DD-scenario depends on metallicity of stars which defines formation time of WD and their mass (see, for instance, Kobayashi et al. (2000)) and on the parameter of common envelopes, since, in crude approximation $a_{f} \propto \alpha_{c e} \lambda a_{0}$, where $a_{0}$ and $a_{f}$ are initial and final separations of components. 
In $10^{10} \mathrm{yr}$ merge the pairs of WD with $a_{f} \sim R_{\odot}$. In the spiral galaxies new pairs of WD form continuously and merger rate continuously increases, since merge both "old" initially relatively wide pairs and "young" relatively close ones. In elliptical galaxies a reservoir of pairs with different combinations of masses of components and separations is created during the burst of star formation and it gradually "melts" and therefore at certain moment after cessation of star formation merger rate starts to decline.

Formation of an accreting CO-WD with initial mass lower than $1.2 M_{\odot}$ and accumulation of $M_{C h}$ by a dwarf require certain time, therefore in semidetached systems SN Ia occur with delay of $\sim 10^{9} \mathrm{yr}$ respective to star formation. Numerous studies of delay time distributions for SN Ia, starting from the papers by Jorgensen et al.(1997) and Yungelson and Livio (2000), show that after a single star-formation burst semidetached systems in which a CO-WD is able to accumulate $M_{C h}$ form and exist over a limited span of time which lasts for several Gyr. In our model, these are systems in which at the instant of Roche-lobe overflow by the donor $M_{w d, 0} \gtrsim 0.85 M_{\odot}$ and $M_{s g, 0} \gtrsim 1.4 M_{\odot}$. Figure 1 shows, that formation rate of semidetached systems with WD able to accumulate $M_{C h}$ even in relatively early stages of galaxy evolution is only a minor fraction of the total formation rate of semidetached systems with WD with surface hydrogen-burning. Semidetached systems with WD form during total lifetime of galaxies evolution, but in the case of burst-like star formation the systems observed at present are not precursors of SN Ia. Currently, in the model galaxy with continuous star formation SN Ia occur in semidetached systems formed approximately $6 \times 10^{8}$ to $2.5 \times 10^{9}$ yrs ago.

Along to the systems in which hydrogen burns stationary, there exist systems in which hydrogen at the surface of WD burns in outbursts. The systems of two types are shown in Fig. 1 separately. For the outbursting systems, we assumed, like in Yungelson et al. (1996) that hydrogen burning time is equal to the time of decline of bolometric luminosity of WD by 3 stellar magnitudes $\left(t_{3 b o l}\right)$. The value of the latter was estimated by interpolation in Prialnik and Kovetz (1995) data. Both systems with stable and unstable hydrogen burning start to form almost simultaneously, but at any time the former dominate, since $t_{3 b o l}$ is short. In the systems with subgiants the fraction of outbursting systems is initially low, since first start to overfill their Roche lobes relatively massive stars with high mass-loss rates. Later, the fraction of systems in which the rate of accretion is lower than the limit for stable hydrogen burning increases since the systems with massive donors finish their evolution fast.

\section{Symbiotic stars}

The second main group of stars with accreting WD are symbiotic stars in which WD are accompanied by giants or supergiants. White dwarfs accrete from the wind of companion. In the initial stage of the evolution of donor along the giant branch stellar wind is weak. In wide systems with orbital periods of hundreds and thousands day accretion initially is extremely inefficient. It takes a long time to accumulate enough hydrogen for the first outburst. Then WD transits into regime of unstable burning and, typically only shortly before the loss of envelope by the giant WD may become a stationary burner (see for details Yungelson et al. (1995) and Lü et al. (2006)). Conventionally, we shall denote as "symbiotic stars" all detached systems in which at least one outburst of nuclear burning at the surface of WD had happened, irrespective to whether such stars correspond to formal phenomenological definition of symbiotic stars.

Only $\sim 0.01 \%$ of all systems of giants and supergiants with WD companions reach the stage of symbiotic star. Duration of the stage of stationary burning of hydrogen at the surface of WD is, as a rule, only $\sim 10^{5} \mathrm{yr}$. At $t=10^{10} \mathrm{yr}$ the "spiral" and "elliptical" model galaxies harbor 1360 and 1260 systems with hydrogen-burning WD, respectively. Currently, about 200 symbiotic stars are known in the Galaxy (Belczynski et al., 2000). Observational estimates based on extrapolation of the number of known objects and estimates of completeness of the observed sample range from $3 \times 10^{3}$ (Allen, 1984) to $3 \times 10^{5}$ (Munari and Renzini, 1992) objects. 


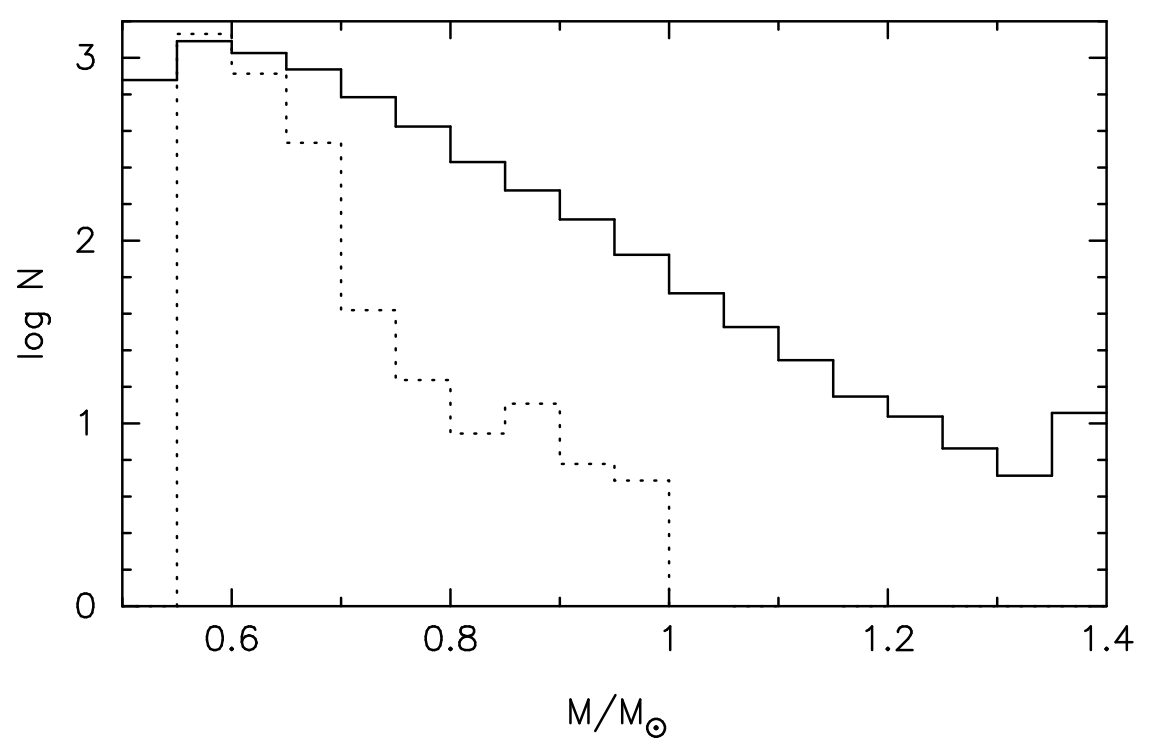

Figure 2. Mass spectrum of accreting WD with nuclear burning at the surface. Solid line - WD in the model system with continuous star formation, dotted line - WD in the model system with burst-like star formation. In both cases we show the sum of the numbers of WD with stationary and nonstationary burning.

As a result of a pointed survey which took into account selection effects, Corradi et al. (2008, 2010) found only 11 new symbiotic star in the sky region where 11 stars were already known before. Thus, it seems that expectations of existence of hundreds of thousands of symbiotic stars in the Galaxy are exaggerated and our estimate of $\sim 1000$ objects is realistic. Time delay of formation of symbiotic stars with respect to star formation is several tens of Myr. This is slightly more than the time of formation of the first WD. Symbiotic stars continue to form for 10 Gyr both in spiral and elliptical galaxies. In the latter case this happens as main-sequence is left by less and less massive stars. As a result, at $t=10^{10} \mathrm{yr}$ the numbers of WD accreting from the wind in the model "spiral" and "elliptical" galaxies are comparable.

Among symbiotic stars about $2 / 3$ of systems are wide, precursors of WD in them did not overflow their Roche lobes. Therefore, mass spectrum of WD in symbiotic stars is dominated by low-mass CO WD $\left(\sim 0.6 M_{\odot}\right)$. Since accretion rates are low, nuclear burning is mostly unstable and in most cases erosion of white dwarfs happens instead of accumulation of mass. Estimates by Lü et al. show that in symbiotic stars during accretion stage mass of WD increases by no more than $\simeq 0.1 M_{\odot}$. This has an important consequence. The masses of new-born $\mathrm{CO}$ WD do not exceed $(1.1-1.2) M_{\odot}$ (Nomoto, 1984; Ritossa et al., 1996; Poelarends et al., 2008). More massive WD are composed by oxygen, neon, and magnesium. Hence, in symbiotic stars CO WD are unable to accumulate $M_{C h}$. Instead of SN Ia accretion-induced collapses (AIC) of ONeMg-WD occur which prduce neutron stars (Nomoto and Kondo, 1991). The rate of AIC's in the model of a spiral galaxy $6.6 \times 10^{-4} \mathrm{yr}^{-1}$ is consistent with the estimate of AIC's in the Galaxy based on the yields of $r$-process elements, if one takes into account that, according to 2-D rotating models of AIC (Fryer et al., 1999; Dessart et al., 2006) only $\sim 0.001 M_{\odot}$ of matter enriched by $r$-process elements is ejected during AIC. In this case, evolution of symbiotic stars results in formation of wide binaries composed by neutron stars and WD. AIC's, themselves, probably explain the weakest peculiar SN Ia (Metzger et al., 2009).

Since binaries with more massive WD complete their evolution faster, in the elliptic galaxies their number must be lower than in spiral ones. This is clearly shown by Fig. 2 , 
in which we render mass spectra of accreting WD in the model galaxies with different star formation history. This circumstance has to influence the number of detectable WD with nuclear burning.

\section{Conclusion}

Above, we presented results of a computation that exemplifies evolution of the number of accreting WD in semidetached binaries with subgiant donors and symbiotic stars and evolution of the SN Ia rate in the galaxies with continuous and burst-like star formation. The following conclusions may be drawn.

1. In both models of galaxies, after $10 \mathrm{Gyr}$ of evolution WD with surface hydrogen burning exist in approximately equal numbers (several thousands, Fig. 1). Test computations show that this result does not change qualitatively if, for instance, other parameters of common envelopes $\left(\alpha_{c e} \lambda=0.5\right.$ or 1$)$, different parameterization of mass-loss rate, Tutukov and Yungelson (1979) equation for common envelopes, different efficiency of matter accumulation are assumed. The number of objects and the rate of SN Ia vary within 30\%3. Using, for instance, a star formation function with an initial burst and a tail extending for several Gyr in the model of elliptical galaxy also would not change results, since, because of short delay times all "old" SN Ia in semidetached systems succeed to explode in 10 Gyr.

Supersoft X-ray sources in elliptical galaxies are observed, but according to model computations among semidetached systems with subgiant donors precursors of SN Ia are absent. In the model of a spiral galaxy such precursors do exist. Potential, but not immediate SN Ia precursors both in spiral and elliptical galaxies may be symbiotic stars if the following conditions are fulfilled. Donor-star has to overfill Roche lobe in the course of evolution and to form a common envelope. In the common envelope separation of WD and the nucleus of the donor must become small enough to enable merger in the future. The sum of the mass of already existing WD and the core of the donor must to exceed $M_{C h}$. As our model computations show, for similar total masses of galaxies, at the age of 10 Gyr the rate of SN Ia in model elliptical galaxy is by 2 orders of magnitude lower than in the spiral one.

2. Every of relatively well studied nearby spiral galaxies (M31, M101, M51, M83) contains about 100 SSS, in nearby elliptical galaxies (NGC4697, NGC4472) their number is several dozens (Di Stefano, 2010a). Taking into account that, according to the estimates of Di Stefano, Chandra is able to observe in these spiral galaxies only objects with mass $\gtrsim(1.0-1.2) M_{\odot}$ and objects with $M \gtrsim 0.8 M_{\odot}$ in elliptical galaxies, the number of observed SSS agrees with expected from observations (Fig. 2).

3. The main scenario for SN Ia in our model is merger of CO WD. In the model spiral galaxy occurrence rate of accumulations of $M_{C h}$ by WD in semidetached systems with subgiant donors at $t=10^{10} \mathrm{yr}$ is by two orders of magnitude lower than merger rate. In the model elliptical galaxy merger becomes a sole mechanism of SN Ia in $\simeq 2.5 \times 10^{9} \mathrm{yr}$ since beginning of star formation. A typical time scale of single-degenerate SN Ia "epoch" of the order of $10^{9} \mathrm{yr}$ is found in practically all studies of delay time distributions of SN Ia (see, e.g., Ruiter et al., (2009), Mennekens et al., (2010) and references therein). For explanation of SN Ia delays up to $\sim 10^{10} \mathrm{yr}$ in SD-scenario it is necessary to assume stabilization of mass-exchange by reemission from the system of a significant fraction of the matter lost by the donor and low specific angular momentum of ejected matter. As a counterargument against this hypothesis may be used the fact that the traces of hydrogen are discovered as yet for only one SN Ia. Even more, almost commonly accepted assumption of possibility of ejection of a significant amount of matter by optically thick wind is put in doubt (Badenes et al., 2007): the wind should result in formation

\footnotetext{
${ }^{3}$ Let note only that for $\alpha_{c e} \lambda=0.5$ at $t=10^{10} \mathrm{yr}$ in the model of elliptical galaxy SN Ia are absent at all.
} 
of cavities in ISM around supernovae remnants which are not observed. If assumption about ejection of excess of matter is incorrect, real occurrence rate of SN Ia in semi-detached systems may be even lower than our estimates.

Despite our model differs from the models of other authors (e.g. Ruiter et al., (2009)) by lower efficiency of accumulation of the matter by WD, qualitative conclusions are similar: most probably, in spiral galaxies both DD- and SD-scenarios work for their total lifetime, but DD-scenario dominates, while in elliptical galaxies only DD-scenario remains efficient in several Gyr after cessation of star formation. Thus, we confirm conclusions made by Jorgensen et al., (1997), Yungelson and Livio (2000), Mennekens et al., (2010).

Let note that lately several close binary WD or their immediate precursors which have $\left(M_{1}+M_{2}\right) \simeq M_{C h}$ and will merge in less than Hubble time were found. These are binary WD 2020-425 ( $P_{\text {orb }}=0.3$ day, $M_{1}+M_{2}=1.348 \pm 0.045 M_{\odot}$, Napiwotzki et al., 2007), a subdwarf accompanied by a white dwarf KPD $1930+2752\left(P_{\text {orb }}=0.37765 \pm 0.00002\right.$ day, $M_{1}+M_{2}=$ $(1.36-1.48) M_{\odot}$, Geier et al., 2007), planetary nebulae nuclei with WD companions TS 01 $\left(P_{\text {orb }}=0.163\right.$ day, $M_{1}=0.54 \pm 0.02 M_{\odot}, M_{2} \approx 0.86 M_{\odot}$, Tovmassian et al., 2010) and V458 Vul $\left(P_{\text {orb }} \approx 0.068\right.$ day, $M_{1} \approx 0.6 M_{\odot}, M_{2} \gtrsim 1.0 M_{\odot}$, Rodríguez-Gil et al., 2010 ).

In the process of CO WD merger, less massive WD transforms into a disk-like structure around more massive companion. It was usually assumed that in this configuration temperature maximum is located at the "disk-dwarf" interface and that carbon burning starts there. In this model burning front propagates inward and transforms a CO WD into an ONeMg one, which collapses with formation of a neutron star. However, Yoon et al., (2007) have shown that under certain conditions the rate of settling of matter from disk onto WD may be low enough for commencing of burning in the center of WD and a SN Ia. The model still must be elaborated but it is quite possible that "theoretical" objections against DD-scenario may be removed. As an additional argument in favor of DD-scenario one may consider discovery of several SN Ia for which the estimates of the mass of ejected radioactive $\mathrm{Ni}$ are close to $M_{C h}$ or even exceed it: SN 2003fg (Howell et al., 2007), SN 2006gz (Hicken et al., 2007), SN 2007if (Scalzo et al., 2010; Yuan et al., 2010), SN 2009dc (Silverman et al., 2010).

4. In the systems with WD accreting from stellar wind, efficiency of matter accumulation by WD is very low. In these systems, according to our computations, only ONeMg WD can accumulate $M_{C h}$. We expect that in such systems evolution should end not by SN Ia, but by accretion-induced collapse and formation of neutron star + WD pairs.

The author acknowledges fruitful discussions with N.N. Chugai, K.A. Postnov, and M.R. Gilfanov. This study was partially supported by RFBR grant 10-02-00231 and by the Program of the Presidium of the Russian Academy of Sciences "Origin and evolution of stars and galaxies".

\section{References}

1. D.A. Allen, Pub. Astron. Soc. Austral. 5, 369 (1984).

2. C. Badenes, J.P. Hughes, E. Bravo, et al., Astrophys. J. 662, 472 (2007).

3. K. Belczyński, J. Mikołajewska, J., U. Munari, et al., Astron. Astrophys. Suppl. Ser. 146, 407 (2000).

4. A. I. Bogomazov, A. V. Tutukov, Astron. Rep, 53, 214 (2009)

5. H. Bondi, Mon. Not. Roy. Astron. Soc. 112, 195 (1952).

6. E. Cappellaro, R. Evans, R. and M. Turatto, Astron. Astrophys. 351, 459 (1999). 
7. R.L.M. Corradi, E.R. Rodríguez-Flores, A. Mampaso, et al., Astron. Astrophys. 480, 409 (2008).

8. R.L.M. Corradi, M. Valentini, U. Munari, et al., Astron. Astrophys. 509, A41 (2010).

9. W. Dehnen and J. Binney, Mon. Not. Roy. Astron. Soc. 294, 429 (1998).

10. L. Dessart, A. Burrows, C. Ott, et al., Astrophys. J. 664, 1063 (2006).

11. R. Di Stefano, Astrophys. J. 712, 728 (2010a).

12. R. Di Stefano, arXiv:1004.1193 (2010b).

13. A.V. Fedorova, A.V. Tutukov, L.R. Yungelson, Astron. Lett 30, 73 (2004).

14. C.L, Fryer, S.E. Woosley, M. Herant, M., et al., Astrophys. J. 520, 650 (1999).

15. S. Geier, S. Nesslinger, U. Heber, et al., Astron. Astrophys. 464, 299 (2007).

16. M. Gilfanov and A. Bogdán, Nature 463, 924 (2010).

17. G. Gilmore, ASPC, 230, 3, 2001.

18. I. Hachisu, M. Kato and K. Nomoto, Astrophys. J. 522, 487 (1999).

19. M. Hicken, P.M. Garnavich, J.L. Prieto, et al., Astrophys. J. 669, L17 (2007).

20. M.S. Hjellming and R.F. Webbink, Astrophys. J. 318, 794 (1987).

21. D.A. Howell, M. Sullivan, P.E. Nugent, et al., Nature 443, 308 (2006).

22. J.R. Hurley, C.A. Tout and O.R. Pols, Mon. Not. Roy. Astron. Soc.329, 897 (2002).

23. I. Iben Jr. and A.V. Tutukov, Astrophys. J. Suppl. Ser. 54, 335 (1984).

24. I. Iben Jr.and A.V. Tutukov, Astrophys. J. Suppl. Ser. 105, 145 (1996).

25. H.E. Jorgensen, V.M. Lipunov, I.E. Panchenko, et al., Astrophys. J. 486, 110 (1997).

26. M. Kato and I. Hachisu, Astrophys. J. 437, 802 (1994).

27. D. Kasen, Astrophys. J. 708, 1025 (2010).

28. C. Kobayashi, T. Tsujimoto and K. Nomoto, Astrophys. J. 539, 26 (2000).

29. Z. T. Kraicheva, E. I. Popova, A. V. Tutukov A. V., et al., SvAL, 7, 269 (1981)

30. V.M. Lipunov and K.A. Postnov, Astrophys. Space. Sci. 145, 1 (1988).

31. G. Lü, L. Yungelson and Z. Han, Mon. Not. Roy. Astron. Soc. 372, 1389 (2006).

32. F. Mannucci, M. Della Valle, N. Panagia, et al., Astron. Astrophys. 433, 807 (2005).

33. N. Mennekens, D. Vanbeveren, J.-P. De Greve et al., Astron. Astrophys. 515, A89 (2010).

34. B.D. Metzger, A.L. Piro, and E.Quataert, Mon. Not. Roy. Astron. Soc. 396, 1659 (2009).

35. U.Munari, A. Renzini, Astrophys. J. 397, L87 (1992).

36. R. Napiwotzki, C.A. Karl, G. Nelemans, et al., ASP Conf. Ser. 372, 387 (2007).

37. G. Nelemans and C.A. Tout, Mon. Not. Roy. Astron. Soc. 356, 753 (2005).

38. G. Nelemans, F. Verbunt, L.R. Yungelson, et al., Astron. Astrophys. 360, 1011 (2000). 
39. G. Nelemans, L.R. Yungelson, S.F. Portegies Zwart, et al., Astron. Astrophys. 365, 491 (2001)

40. K. Nomoto, Astrophys. J. 277, 791 (1984).

41. K. Nomoto and Y. Kondo, Astrophys. J. 367, L19 (1991).

42. F. Patat, P. Chandra, R. Chevalier. et al., Science, 317, 924 (2007).

43. D. Prialnik and A. Kovetz, Astrophys. J. 445, 789 (1995).

44. A.J.T. Poelarends, F. Herwig, N. Langer, et al., Astrophys. J. 675, 614 (2008).

45. A. J. Ruiter, K. Belczynski and C. Fryer, Astrophys. J. 699, 2026 (2009).

46. C. Ritossa, E. Garcia-Berro and I. Iben Jr., Astrophys. J. 460, 489 (1996).

47. P. Rodríguez-Gil, M. Santander-García, C. Knigge, et al., arXiv1006.1075 (2010).

48. J.M. Silverman, M. Ganeshalingam, W. Li, et al., arXiv:1003.2417 (2010).

49. R.A. Scalzo, G. Aldering, P. Antilogus, et al., Astrophys. J. 713, 1073 (2010).

50. G. Tovmassian, L. Yungelson, T. Rauch, et al., Astrophys. J. 714, 178 (2010).

51. J.W. Truran and A.G.W. Cameron, Astrophys. Space. Sci. 14, 179 (1971).

52. A.V. Tutukov and L. Iungelson, IAU Symp. 83, 401 (1979).

53. A.V. Tutukov and L. Yungelson, Mon. Not. Roy. Astron. Soc. 268, 871 (1994).

54. A.V. Tutukov and L.R. Yungelson, Astron. Rep. 46, 667 (2002).

55. J. Whelan and I. Iben Jr., Astrophys. J. 186, 1007 (1973).

56. E.P.J. van den Heuvel, D. Bhattacharya, K. Nomoto, et al., Astron. Astrophys. 262, 97 (1992).

57. S. Vereshchagin, А. Тутуков, Л. Юнгельсон et al., Astrophys. Space. Sci., 142, 245 (1988).

58. N. Vranesevic, R.N. Manchester, D.R. Lorimer et al., Astrophys. J., 617, L139 (2004).

59. R.F. Webbink, Astrophys. J. 277, 355 (1984).

60. C.-S. Yoon, P. Podsiadlowski and S. Rosswog, Mon. Not. Roy. Astron. Soc. 380, 933 (2007).

61. F. Yuan, R.M. Quimby, J.C. Wheeler, et al., arXiv:1004.3329 (2010).

62. L.R. Yungelson, M. Livio, J.W. Truran, et al., Astrophys. J. 466, 890 (1996).

63. L.R. Yungelson, M. Livio, A. Tutukov, et al.,. Astrophys. J. 447, 656 (1995).

64. L.R. Yungelson and M. Livio, Astrophys. J. 497, 168 (1998).

65. L.R. Yungelson and M. Livio, Astrophys. J. 528, 108 (2000). 